\title{
Relação entre artrite idiopática juvenil e disfunção temporomandibular: uma revisão
} de literatura

\author{
Relationship between juvenile idliopathic arthritis and temporomandibular disorders: a literature \\ review
}

Relación entre la artritis idiopática juvenil y el trastorno temporomandibular: una revisión de la literatura

Recebido: 04/03/2021 | Revisado: 11/03/2021 | Aceito: 12/03/2021 | Publicado: 19/03/2021

Lucas Oliveira Macêdo ORCID: https://orcid.org/0000-0002-4920-4878 Universidade Federal da Paraíba, Brasil E-mail: lucasmacedo002@gmail.com

Ery Jeverson Tolentino Severo ORCID: https://orcid.org/0000-0002-0118-4046 Universidade Federal da Paraíba, Brasil E-mail: jeverson59@hotmail.com

Josinaldo Maria da Silva Filho

ORCID: https://orcid.org/0000-0003-0840-9612 Universidade Federal da Paraíba, Brasil E-mail: josinaldo.filho@live.com

Rejane Targino Soares Beltrão ORCID: https://orcid.org/0000-0002-3962-1053 Universidade Federal da Paraíba, Brasil E-mail: rejanetsb@uol.com.br

Robinsom Viégas Montenegro ORCID: https://orcid.org/0000-0002-8253-5361 Universidade Federal da Paraíba, Brasil E-mail: rvmontenegro@hotmail.com André Ulisses Dantas Batista

ORCID: https://orcid.org/0000-0003-1593-0174 Universidade Federal da Paraíba, Brasil E-mail: andreulisses@yahoo.com.br

\section{Resumo}

O objetivo deste artigo foi realizar uma revisão sobre Artrite Idiopática Juvenil (AIJ), destacando o comprometimento das articulações temporomandibulares (ATMs) e as consequências orofaciais dessa patologia, incluindo sua possível relação com sinais e sintomas de Disfunções Temporomandibulares (DTMs), assim como as abordagens terapêuticas indicadas. Foi realizada uma busca de artigos nas bases de dados "Pubmed" e "SciELO" de novembro de 2018 a janeiro de 2019 utilizando os seguintes descritores: «temporomandibular joint (TMJ) AND arthritis», «Juvenile idiopathic arthritis (JIA) AND treatment AND JIA» e «orofacial AND manifestations AND JIA» e seus respectivos em português. Os critérios de inclusão foram: artigos que descrevessem a AIJ na ATM, com ênfase no diagnóstico, consequências e no tratamento. Os critérios de exclusão se deram por relevância bibliográfica, artigos com publicações anteriores ao ano de 2012 e artigos sem acesso integral ao conteúdo. Obteve-se 100 artigos completos, dos quais 21 foram utilizados como base científica apropriada, após análise e seleção e obedecendo aos critérios de inclusão e exclusão. Foi adicionado um livro considerado relevante para a pesquisa. A AIJ atinge constantemente as articulações temporomandibulares causando vários tipos de alterações orofaciais e disfunções nessa articulação. $\mathrm{O}$ diagnóstico precoce é difícil, porém de suma importância, visto que visa minimizar complicações futuras, tanto a nível oclusal, quanto a interferências no crescimento normal da mandíbula. O diagnóstico diferencial e precoce entre DTM e AIJ é de extrema importância, pois servirá como base para escolha do tratamento mais adequado para esses pacientes.

Palavras-chave: Artrite idiopática juvenil; Articulação temporomandibular; Síndrome da disfunção da articulação temporomandibular; Reabilitação; Diagnóstico.

\footnotetext{
Abstract

The aim of this article was to conduct a review on Juvenile Idiopathic Arthritis (JIA), highlighting the impairment of the temporomandibular joints (TMJs) and the orofacial consequences of this pathology, including its possible
} 
relationship with signs and symptoms of Temporomandibular Disorders (TMDs), as well as the indicated therapeutic approaches. A search for articles was carried out in the "Pubmed" and "SciELO" databases from November 2018 to January 2019 using the following descriptors: «temporomandibular joint (TMJ) AND arthritis», «Juvenile idiopathic arthritis (JIA) AND treatment AND JIA »and« orofacial AND manifestations AND JIA »and their respective in Portuguese. Inclusion criteria were articles describing JIA in the TMJ, with an emphasis on diagnosis, consequences and treatment. The exclusion criteria were given by bibliographic relevance, articles with publications prior to 2012 and articles without full access to the content. Hundred complete articles were obtained, of which 21 were used as an appropriate scientific basis, after analysis and selection and obeying the inclusion and exclusion criteria. A book was considered relevant to the research. JIA constantly affects the temporomandibular joints causing various types of orofacial changes and dysfunctions in that joint. Early diagnosis is difficult, but of paramount importance, as it aims to minimize future complications, both at the occlusal level, as well as interfering with the normal growth of the mandible. The differential and early diagnosis between TMD and JIA is extremely important, as it will serve as a basis for choosing the most appropriate treatment for these patients.

Keywords: Juvenile idiopathic arthritis; Temporomandibular joint; Temporomandibular joint dysfunction syndrome; Rehabilitation; Diagnosis.

\section{Resumen}

El objetivo de este artículo fue realizar una revisión sobre la artritis idiopática juvenil (AIJ), destacando el deterioro de las articulaciones temporomandibulares (ATM) y las consecuencias orofaciales de esta patología, incluida su posible relación con los signos y síntomas de los trastornos temporomandibulares (TTM), así como los enfoques terapéuticos indicados. Se realizó una búsqueda de artículos en las bases de datos "Pubmed" y "SciELO" desde noviembre de 2018 hasta enero de 2019 utilizando los siguientes descriptores: «temporomandibular joint (TMJ) AND arthritis», «Juvenile idiopathic arthritis (JIA) AND treatment AND JIA» y «orofacial AND manifestations AND JIA» y sus respectivos en portugués. Los criterios de inclusión fueron: artículos que describían la AIJ en la ATM, con énfasis en el diagnóstico, las consecuencias y el tratamiento. Los criterios de exclusión fueron dados por relevancia bibliográfica, artículos con publicaciones anteriores a 2012 y artículos sin acceso completo al contenido. Se obtuvieron 100 artículos completos, de los cuales 21 se utilizaron como una base científica apropiada, después del análisis y la selección y obedeciendo a los criterios de inclusión y exclusión. Un libro fue considerado relevante para la investigación. La AIJ afecta constantemente las articulaciones temporomandibulares causando varios tipos de alteraciones orofaciales y disfunciones en esa articulación. El diagnóstico precoz es difícil, pero de suma importancia, ya que tiene como objetivo minimizar las complicaciones futuras, tanto a nivel oclusal, como interferir con el crecimiento normal de la mandíbula. El diagnóstico diferencial y temprano entre los TMM y AIJ es extremadamente importante, ya que servirá como base para elegir el tratamiento más adecuado para estos pacientes.

Palabras clave: Artritis idiopática juvenil; Articulación temporomandibular; Síndrome de la disfunción de Articulación temporomandibular; Rehabilitación; Diagnóstico.

\section{Introduçãa}

A artrite idiopática juvenil (AIJ) é uma doença pediátrica crônica corriqueira na infância e na adolescência, compreendendo um grupo de entidades clínicas distintas de etiologia desconhecida (Carvalho et al., 2012; Maliki \& Sztajnbok, 2016). Essa doença é caracterizada por uma inflamação crônica das articulações que persiste por pelo menos 6 semanas, com início antes dos 16 anos de idade (Carvalho et al., 2012; Pinto et al., 2018).

Atualmente, a AIJ é classificada, segundo a International League of Associations for Rheumatology (ILAR), como: artrite sistêmica, poliartrite (Fator Reumatoide negativo), poliartrite (Fator Reumatoide positivo), oligoartrite (persistente ou estendida), artrite relacionada com entesite, artrite psoriásica e indiferenciada (Carvalho et al., 2012; Maliki \& Sztajnbok, 2016; Pinto et al., 2018), baseando-se no número de articulações afetadas, tempo de duração, presença de marcadores sorológicos, e até mesmo a idade de início da doença.

Apesar de muitos estudos, a etiologia ainda é incerta, considerando-se infecções, traumas físicos, susceptibilidade genética e distúrbios imunológicos como fatores importantes (Pinto et al., 2018). Pacientes diagnosticados com disfunção temporomandibular (DTM), correlacionado a AIJ, apresentam alterações com variados níveis de gravidade no sistema estomatognático.

Essas alterações podem levar a um prejuízo do crescimento mandibular, acarretando em mudanças orofaciais importantes para a vida adulta do paciente com AIJ, que podem ter impacto direto na qualidade de vida desses indivíduos 
(Carvalho et al., 2012). O diagnóstico precoce de distúrbios de crescimento facial e disfunções da articulação temporomandibular (ATM) parece minimizar problemas dentários futuros em pacientes com AIJ e aumentar o sucesso do tratamento dessas alterações nesses indivíduos (Carvalho et al., 2012).

Durante o processo deletério, a ATM pode ser afetada pela AIJ, resultando em debilitação mandibular, incluindo capacidade mastigatória reduzida, má oclusão, micrognatia e modificações na anatomia facial que podem ser oriundas de um distúrbio de crescimento associado a danos condilares (Pinto et al., 2018). Como a ATM é um centro de crescimento secundário para a face, o reconhecimento precoce do seu envolvimento pela AIJ é de fundamental importância para minimizar os efeitos adversos de crescimento facial e inflamação persistente da ATM (Granquist, 2018).

A ressonância magnética é considerada o padrão ouro para identificação de mudanças na estrutura da ATM (Maliki \& Sztajnbok, 2016; Montesinos et al., 2018), a qual pode detectar de forma precoce anormalidades ou destruição articular, como as erosões ósseas e sinovites. O diagnóstico deve ser baseado na história médica e odontológica, apresentação clínica e alterações nos exames de imagem e laboratoriais (Abramowicz, Levy, Prahalad, Travers, \& Angeles-Han, 2019; Maliki \& Sztajnbok, 2016).

O objetivo deste artigo é fazer uma revisão sobre AIJ, destacando o comprometimento das ATMs e as consequências orofaciais nesses pacientes, incluindo a relação entre fatores hereditários e os sinais e sintomas da AIJ e das DTMs, buscandose verificar uma possível relação entre os problemas e esclarecendo aos cirurgiões-dentistas quanto a importância do diagnóstico precoce dessas alterações.

\section{Metodologia}

O presente trabalho trata-se de um estudo de natureza qualitativa (Pereira et al., 2018). Assim, foi realizada uma busca de artigos nas bases de dados "Pubmed" e "SciELO" de novembro de 2018 a janeiro de 2019 utilizando os seguintes descritores: «temporomandibular joint (TMJ) AND arthritis», «Juvenile idiopathic arthritis (JIA) AND treatment AND JIA» e «Orofacial AND manifestations AND JIA» e seus respectivos em português. Os critérios de inclusão foram: artigos que descrevessem a AIJ na ATM, com ênfase nas consequências, diagnóstico e tratamento escritos em inglês ou português, com resumo disponível na base de dados e acesso integral ao artigo. Foram excluídos os artigos com publicações anteriores ao ano de 2012, artigos incompletos e de baixa relevância. Dos 100 artigos obtidos, 21 foram utilizados como base para essa revisão atualizada, obedecendo os critérios de inclusão e exclusão.

A seleção dos artigos foi realizada sequencialmente pelo título, resumo e então leitura completa do mesmo de modo que em cada etapa foi verificada a inclusão do artigo pela sua compatibilidade com o tema em questão.

Após a leitura dos textos, os mesmos deram base à avaliação e discussão do tema através da análise dos seguintes aspectos: patogênese, epidemiologia, etiologia, características clínicas, diagnóstico, tratamento e a relação entre AIJ e sinais e sintomas de DTM.

\section{Análise e Integração da Informação}

\section{Patogênese}

A patogênese ainda é pouco conhecida, contudo o fator genético (congênito ou hereditário) tem sido considerado um dos mais significativos para a manifestação, manutenção e aumento da frequência, precipitando o processo autoimune da AIJ (Pinto et al., 2018).

O processo de comprometimento da ATM pela AIJ inicia-se na membrana sinovial, em decorrência de alterações inflamatórias, que resulta em sinovite. A perpetuação deste processo inflamatório leva à liberação de mediadores químicos (IL1 e TNF- $\alpha$ ), durante a fase ativa da doença, que por sua vez atraem mais células inflamatórias, causando liberação de enzimas que fragmentam colágeno e proteínas de matriz com consequente destruição articular (Carvalho et al., 2012). 
Com a destruição articular, forma-se um tecido de granulação que substitui o tecido destruído. Esse tecido, por sua vez, tem poder de penetração na cartilagem articular e no osso subcondral, formando o pannus articular, o que causa destruição dos côndilos (Carvalho et al., 2012; Niibo et al., 2016), levando a alterações faciais na maioria dos casos.

\section{Epidemiologia}

A AIJ afeta até 150 em 100.000 crianças em todo o mundo (Del Giudice, Swart, \& Wulffraat, 2017; Hügle et al., 2017; Niibo et al., 2016). O início da doença, na maioria das vezes, cursa entre 4 anos de idade ou nos anos imediatamente precedentes à puberdade (Leksell, Hallberg, Horne, Ernberg, \& Hedenberg-Magnusson, 2017).

A ATM é uma das articulações mais comumente afetadas, apresentando prevalência de $17 \%$ a $87 \%$ dos casos (Chatzigianni, Kyprianou, Papadopoulos, \& Sidiropoulou, 2018; Del Giudice et al., 2017; Klenke et al., 2018). O acometimento bilateral das ATMs ocorre em 30\% dos pacientes. Já em 22\% dos acometidos, o envolvimento é unilateral (Klenke et al., 2018).

A AIJ é geralmente mais comum em mulheres (Niibo et al., 2016), sendo os subtipos oligoarticular e poliarticular (Fator Reumatóide positivo: 3\% a 10\% e Fator Reumatóide negativo: 25\%), respectivamente, mais encontrados dentre os pacientes diagnosticados com AIJ.

\section{Etiologia}

A AIJ é uma patologia multifatorial influenciada por fatores ambientais, genéticos e hereditários (Niibo et al., 2016). Outra possível etiologia sugerida tem sido micro traumas repetitivos dos componentes articulares, infecções sistêmicas, desordens congênitas ou idiopáticas.

Os fatores ambientais podem provocar desenvolvimento da autoimunidade por ativação do sistema imunitário, assim sendo, vários agentes infeciosos têm sido relacionados com o início da doença (Rigante, Bosco, \& Esposito, 2015).

O stress, os fatores psicológicos e hábitos tabagistas maternos são relatados como fatores de risco no início e na exacerbação da AIJ. O stress leva ao aumento da IL-6, uma citocina importantíssima desta patologia, este é mais importante considerar na idade adulta do que na infância (Rigante et al., 2015).

Dados sugerem que fatores genéticos contribuem substancialmente para a predisposição da AIJ. Os genes positivos na AIJ parecem ter efeito em uma época, durante a qual, podem contribuir para o risco de doença. O subgrupo que se identifica mais com este acontecimento é o oligoarticular (Rigante et al., 2015).

\section{Características clínicas}

A AIJ é uma doença autoimune que afeta as articulações e que pode causar inflamação sinovial crônica com alto risco de destruição progressiva das ATMs, levando a importantes alterações funcionais, como: redução da mobilidade e disfunção mandibular, distúrbios dos músculos da mastigação, variáveis graus de dor orofacial e dimorfismo facial, envolvimento de outras articulações do corpo, além dos sintomas gerais (sistêmicos), como: febre, edema nas articulações e dor (Carvalho et al., 2012; Niibo et al., 2016; Piancino et al., 2018; Rongo et al., 2019).

Lesões erosivas da região condilar e fechamento prematuro da epífise óssea devido ao uso prolongado de antiinflamatórios, promovem uma deficiência de crescimento mandibular, uma vez que a região condilar é sabidamente o principal centro de crescimento dessa região (Carvalho et al., 2012; Pinto et al., 2018). O subtipo de AIJ também influência na gravidade das alterações orofaciais. No tipo poliarticular, tais alterações são mais pronunciadas quando comparadas às de outros subtipos; o tempo de início da doença tem forte influência no crescimento anormal da mandíbula (Carvalho et al., 2012).

As manifestações articulares características da AIJ são DTMs, inchaço (edema) da articulação, artralgia e mobilidade articular reduzida. 
Durante o exame clínico na AIJ, é muito comum detectar-se uma limitação marcante de abertura de boca, principalmente se a doença está na fase ativa, micrognatia, rotação posterior da mandíbula, retrognatia, mordida aberta anterior, má oclusões (Chatzigianni et al., 2018; Del Giudice et al., 2017; Pinto et al., 2018), além da dor, em repouso ou função, ou limitação de movimentos, crepitações e edema por pelo menos 6 semanas (Abramowicz et al., 2019; Klenke et al., 2018; Leksell et al., 2017; Rongo et al., 2019). A rigidez matinal da ATM também é uma queixa frequente (Niibo et al., 2016; Stoustrup et al., 2012). Dor e estalido são os sintomas mais comuns relatados pelos pacientes (Montesinos et al., 2018).

A mordida aberta pode resultar em sobrecarga mastigatória dos dentes posteriores, músculos e estruturas articulares, o que pode causar desequilíbrios musculares e distúrbios intracapsulares (Niibo et al., 2016), acarretando na exacerbação da dor na área da ATM e na região massetérica.

Geralmente, o envolvimento da ATM começa unilateralmente e torna-se bilateral à medida que a doença se agrava (Farronato et al., 2009). A assimetria facial é decorrente do acometimento unilateral da ATM ou quando há o envolvimento bilateral desproporcional das ATMs (Chatzigianni et al., 2018). A retrognatia leva a um padrão de classe II, deixando o paciente com um perfil facial convexo.

Se o acometimento é bilateral, a hipoplasia mandibular causa repercussão estética facial com uma característica relatada e conhecida como "face de pássaro" (Del Giudice et al., 2017; Farronato et al., 2009).

Segundo os estudos de Chatzigianni et al. (Chatzigianni et al., 2018), que relacionou as características oclusais ao gênero e afecções condilares, concluiu-se que a maioria das crianças, tanto do sexo masculino quanto feminino, sem lesões condilares apresentavam oclusão em Classe I. Em contraste, a má oclusão de classe II estava presente na maioria dos pacientes que apresentaram algum tipo de dano condilar, unilateral ou bilateral.

A diminuição do comprimento do arco inferior e o maior índice de irregularidade nos incisivos inferiores em crianças com acometimento condilar bilateral, em comparação ao grupo não condicionado, implicam no importante papel da ATM, não apenas na forma craniofacial, mas também na arcada dentária (Chatzigianni et al., 2018).

\section{Diagnóstico}

O envolvimento da ATM na AIJ muitas vezes é difícil de detectar, podendo esta ser assintomática (Klenke et al., 2018; Kovalko, Stoustrup, \& Twilt, 2018). O prognóstico desses pacientes é variável, estando comumente relacionado a prevalência de suas fases aguda ou crônica, tempo de início, o subtipo da AIJ desenvolvido, gravidade do envolvimento articular e a idade do paciente (Farronato et al., 2009).

O diagnóstico é baseado em uma combinação da história médica, apresentação clínica e anormalidades radiológicas e laboratoriais (Abramowicz et al., 2019; Kovalko et al., 2018). É altamente recomendável acompanhar a função mastigatória de pacientes com AIJ, além da capacidade funcional da ATM (Carvalho et al., 2012; Kovalko et al., 2018). Alguns autores apontam sinais e sintomas de DTM como manifestações iniciais da AIJ ou as únicas manifestações (Pinto et al., 2018), e os achados iniciais mais comumente encontrados na ressonância magnética (RM) são as sinovites e efusões.

De acordo com os estudos de Stoustrup e colaboradores, todos os pacientes apresentaram dor orofacial, com localização, na maioria dos casos, na área da ATM e região do músculo masseter, principalmente durante a mastigação, abertura máxima da boca (Stoustrup et al., 2012). Devido seu caráter autoimune, provoca rápida destruição condilar acarretando em alterações oclusais e faciais.

Alterações patomorfológicas típicas presentes na ATM de pacientes com AIJ são achatamento condilar, osteófitos, erosões e esclerose. A assimetria de toda a mandíbula também é descrita (Klenke et al., 2018).

Um diagnóstico precoce da artrite da ATM é essencial para diminuir a destruição condilar. No momento em que os sinais clínicos e morfológicos, como o retrognatismo ou a assimetria mandibular, tornam-se óbvios, os côndilos já estão 
irreversivelmente danificados (Niibo et al., 2016).

Existem alguns achados de imagem que correlacionam sinais e sintomas clínicos que podem ajudar a estabelecer um diagnóstico precoce da artrite da ATM. A avaliação clínica da capacidade limitada de abertura da bucal, desvio mandibular durante a abertura e redução da translação condilar são alguns dos indicadores clínicos (Klenke et al., 2018).

Atualmente, a RM é o exame mais confiável para uma avaliação objetiva e não invasiva do tecido mole intracapsular (Kovalko et al., 2018; Montesinos et al., 2018). É considerada a melhor forma de diagnóstico precoce, uma vez que pode detectar alterações musculares, sinoviais cartilaginosas e ósseas, mesmo em início de progressão (Montesinos et al., 2018), possibilitando a intervenção terapêutica o mais cedo possível e minimizando os danos orofaciais aos pacientes.

No entanto, devido ao fato de que derrames da ATM também podem ser encontrados em crianças sem AIJ e sem danos na ATM, o risco de super-diagnosticar danos na ATM é substancial (Rongo et al., 2019).

A tomografia computadorizada (TC) desempenha importante papel no diagnóstico de anomalias ósseas e condições patológicas da ATM, proporcionando uma visualização completa da região articular e um diagnóstico mais preciso (dos Santos Silveira et al., 2014).

A TC é um exame de imagem superior ao exame de imagem convencional para ATM, pois permite uma visualização tridimensional adequada e precisa de todos os detalhes do osso, sem a limitação de sobreposição, proporcionando o real tamanho e formato das estruturas anatômicas, alterações morfológicas e condições patológicas (dos Santos Silveira et al., 2014).

A ultrassonografia fica bastante limitada pela anatomia da região da ATM, sendo pouco utilizada. A radiografia panorâmica só possibilita a visualização de lesões tardiamente, quando as erosões condilares já estão instaladas, além de forte sobreposição de imagens.

No que diz respeito aos exames laboratoriais, os biomarcadores de doenças são ferramentas valiosas para o diagnóstico correto e para a avaliação de protocolos de tratamento apropriados (Niibo et al., 2016). O uso de biomarcadores confiáveis seria benéfico para fins de diagnóstico ou para monitorar o curso da doença.

Vários biomarcadores já estão em uso difundido na rotina de tratamento de AIJ, como anticorpo antinuclear (ANA), proteína C-reativa (PCR) e fator reumatóide (FR). Duas formas poliarticulares da AIJ são diferenciadas pela ausência ou presença de autoanticorpo sérico, conhecido como fator reumatóide (FR) (Niibo et al., 2016). A PCR permite identificação de grandes alterações sanguíneas, sendo necessário rápida indicação ao reumatologista.

\section{Tratamento}

O principal objetivo do tratamento da AIJ é reduzir a dor do indivíduo, prevenir ou controlar o dano articular e a perda de função, além de assegurar um crescimento simétrico e diminuir as alterações orofaciais e dentoaoveolares, garantindo remissão da atividade da doença e normalizar a função motora.

Atenção particular deve ser dada ao tratamento correto dos côndilos lesados, para diminuir os danos funcionais e estéticos em pacientes com AIJ. O diagnóstico precoce e a observação por equipes multidisciplinares, composta por ortodontistas, cirurgiões bucomaxilofaciais, reumatologistas, radiologistas e pediatras, é necessária para o manejo clínico e abrangente desses pacientes (Chatzigianni et al., 2018; Stoustrup et al., 2012).

O manejo das complicações da ATM em pacientes com a AIJ baseia-se em uma combinação de aconselhamentos, terapias farmacológicas, fisioterapia, aparelhos oclusais, ortodontia e cirurgia (em casos mais severos de destruição) (Kovalko et al., 2018; Niibo et al., 2016; Stoustrup et al., 2012).

O tratamento da artrite com envolvimento da ATM inclui três aspectos distintos: (1) o tratamento da inflamação ativa com medicação local ou sistêmica, (2) tratamento dos sinais e/ou sintomas induzidos pela artrite (por exemplo, estabilização da dor), e (3) tratamento do desvio do crescimento dentofacial com dispositivos ortopédicos ou cirurgia (Kovalko et al., 2018). 
Do ponto de vista farmacológico, pode-se lançar mão do uso de drogas anti-inflamatórias não esteroidais (AINEs), drogas antirreumáticas modificadoras da doença (DMARDs), agentes biológicos, esteroides intra-articulares ou orais, Metotrexato e Etanercept (Del Giudice et al., 2017).

Os AINEs aliviam a dor e reduzem a inflamação, mas não previnem os danos às articulações. Os corticosteroides intraarticulares (injeções que consistem tipicamente em hexacetonido de triancinolona a uma dose de 5 a $20 \mathrm{mg}$ /articulação) oferecem um alívio muito rápido dos sintomas e podem poupar a necessidade de terapia sistêmica. Os corticosteroides intra-articulares podem ser benéficos nos casos em que a artrite ativa da ATM persista apesar do uso de terapia sistêmica (Kovalko et al., 2018; Niibo et al., 2016).

Uma preocupação é o risco de formação óssea heterotópica (HBF), definida como a presença de osso em tecidos moles, tal como dentro ou fora da cápsula da ATM. Isto pode ocorrer mesmo nas articulações periféricas, onde foram notificadas calcificações periarticulares ou intra-articulares após injeções de corticosteroides em 0,2 a $6 \%$ das articulações injetadas (Kovalko et al., 2018; Stoll et al., 2018).

A ossificação periarticular após a terapia intra-articular é composta de cristais de hidroxiapatita que podem produzir DTM (Stoll et al., 2018).

O metotrexato continua a ser a DMARD mais utilizada na gestão da AIJ por possuir efeitos benéficos de longo prazo no controle da atividade da doença moderada à grave, geralmente associado a AINEs ou corticoides. Em pacientes resistentes a essa medicação, pode ser indicado outro DMARD ou agentes biológicos que tenham como alvo o fator de necrose tumoral (TNF), interleucina (IL) -1 e IL-6 (Stoor, Hodzic, \& Arte, 2018).

No entanto, o metotrexato pode estar associado a uma série de efeitos adversos potencialmente graves e, portanto, seu uso requer monitoramento cuidadoso com exames de sangue e supervisão clínica de um especialista médico (Niibo et al., 2016).

O paciente deve, portanto, ser instruído em exercícios de auto-administração que se encaixam em quatro tipos: alongamento muscular passivo, alongamento muscular assistido, exercícios de resistência e treinamento postural. Estes exercícios são repetidos dez vezes, seis sessões por dia. Se algum deles provocar dor, eles são descontinuados. O principal objetivo desses exercícios é ajudar a restaurar a função normal e amplitude de movimentos (Niibo et al., 2016).

Do ponto de vista da ortodontia a importância do tratamento precoce com um aparelho ortopédico funcional é demonstrada, não apenas para prevenir deformidades e danos funcionais, mas também para mitigar os sintomas subjetivos durante uma fase aguda da doença, mal controlada apenas pela terapia farmacológica. $\mathrm{O}$ tratamento ortopédico é muito importante, pois melhora a sinovite e a dor do paciente, por causar a descompressão na região posterior e pode em alguns casos evitar a necessidade do tratamento cirúrgico (Farronato et al., 2009; Kovalko et al., 2018; Stoor et al., 2018).

Durante a fase ativa, o aparelho ortopédico protege a ATM dos danos dos efeitos prejudiciais da sinovite na superfície articular por meio da descompressão condilar. Durante a fase de remissão, o ativador tem uma função ortopédica, modificando o padrão de crescimento por alongamento muscular além da posição de repouso (Farronato et al., 2009; Kovalko et al., 2018).

A terapia ortopédica melhora o potencial de crescimento esquelético através da tração na superfície condilar pelas fibras cartilaginosas, causando aumento condilar ao longo do tempo. Tendo em vista que os pacientes são jovens, o tratamento começa durante um período acelerado de crescimento esquelético, antes do surto de crescimento puberal, sendo o período mais favorável para usar um aparelho funcional (Farronato et al., 2009).

$\mathrm{Na}$ fase de remissão e na fase aguda, redireciona-se o crescimento condilar, modificando o posicionamento condilar que se encontra bloqueado pelo processo inflamatório, assim, o paciente tende a viver sem desenvolver deformações sérias, podendo melhorar os aspectos estéticos e funcionais.

O tratamento cirúrgico é usado para corrigir alterações craniofaciais severas e não fazem parte do tratamento real da artrite ativa da ATM. Esses procedimentos geralmente não são realizados até que os pacientes tenham atingido a idade adulta, 
devido ao crescimento mandibular contínuo até os 25 anos. No entanto, o tratamento das deformidades dentofaciais induzidas por artrite grave com distrações cirúrgicas mandibulares mostrou ser uma opção de tratamento segura e previsível (Del Giudice et al., 2017; Kovalko et al., 2018). A cirurgia ortognática, distração osteogênica do ramo mandibular e as reconstruções articulares com próteses funcionais (próteses condilares), são algumas das modalidades cirúrgicas bastante utilizadas na correção de defeitos graves (Stoor et al., 2018).

\section{Importância do diagnóstico diferencial entre DTM articular e AIJ}

O diagnóstico diferencial mostra-se extremamente necessário à obtenção correta do diagnóstico final de uma doença. Através dele é possível verificar qual a melhor maneira de se tratar o indivíduo doente através do uso correto de medicamentos ou outras modalidades de tratamento.

As desordens articulares inflamatórias são caracterizadas por uma dor profunda contínua, geralmente acentuada com a função. A osteoartrite é um processo degenerativo considerado uma resposta do organismo ao aumento da carga na articulação. Isso causa erosão óssea com consequente evidência radiográfica de osteoartrite. É importante salientar que essas alterações são vistas apenas em estágios avançados da doença (Okeson, 2011). Contudo, uma vez que a carga diminui, a condição artrítica pode se tornar adaptativa, ainda que a morfologia óssea continue alterada. Essa condição adaptativa é chamada de osteoartrose (Okeson, 2011).

A osteoartrite causa a presença de sons articulares (crepitações) à palpação durante o movimento articular, artralgia unilateral que piora no final do dia e limitação de abertura bucal. Geralmente está associada ao deslocamento de disco sem redução, acelerando o processo destrutivo, já a osteoartrose apresenta ruídos articulares quando em função, porém sem sintomatologia dolorosa. O diagnóstico é feito a partir da história e exame clínico do paciente e exames de imagem da ATM (Okeson, 2011).

\section{Conclusão}

A AIJ atinge frequentemente as ATMs causando vários tipos de alterações orofaciais e disfunção nessa articulação. O diagnóstico precoce é difícil, porém de suma importância, tendo a equipe multidisciplinar papel fundamental no correto tratamento desses pacientes, a partir das diferentes modalidades terapêuticas empregadas, visando minimizar complicações futuras que interferem no crescimento normal da mandíbula, quanto a nível oclusal. O diagnóstico diferencial entre DTM e AIJ é de extrema importância, pois ele irá servir como base para escolha do tratamento mais adequado para esses pacientes.

Contudo, estudos posteriores são necessários em busca de novas informações sobre a AIJ, principalmente em relação a etiologia, o que poderá proporcionar tratamentos mais eficazes e viáveis, minimizando os efeitos ao longo e melhorando a qualidade de vida do paciente.

\section{Referências}

Abramowicz, S., Levy, J. M., Prahalad, S., Travers, C. D., \& Angeles-Han, S. T. (2019). Temporomandibular joint involvement in children with juvenile idiopathic arthritis: a preliminary report. Oral Surgery, Oral Medicine, Oral Pathology and Oral Radiology, 127(1), 19-23.

Carvalho, R. T. de, Braga, F. S. F. F., Brito, F., Capelli Junior, J., Figueredo, C. M., \& Sztajnbok, F. R. (2012). Alterações da articulação temporomandibular e suas repercussões orofaciais em pacientes portadores de artrite idiopática juvenil. Revista Brasileira de Reumatologia, 52(6), 907-911.

Chatzigianni, A., Kyprianou, C., Papadopoulos, M. A., \& Sidiropoulou, S. (2018). Dentoalveolar characteristics in children with juvenile idiopathic arthritis. Journal of Orofacial Orthopedics/Fortschritte Der Kieferorthopadie, 79(2), 133-139.

Del Giudice, E., Swart, J. F., \& Wulffraat, N. M. (2017). Juvenile Idiopathic Arthritis. In Comorbidity in Rheumatic Diseases, 69, 265-288, Cham: Springer International Publishing.

Farronato, G., Carletti, V., Maspero, C., Farronato, D., Giannini, L., \& Bellintani, C. (2009). Craniofacial growth in children affected by juvenile idiopathic arthritis involving the temporomandibular joint: Functional therapy management. Journal of Clinical Pediatric Dentistry, 33(4), 351-357. 
Granquist, E. J. (2018). Treatment of the Temporomandibular Joint in a Child with Juvenile Idiopathic Arthritis. Oral and Maxillofacial Surgery Clinics of North America, 30(1), 97-107.

Hügle, B., Spiegel, L., Hotte, J., Wiens, S., Herlin, T., Cron, R. Q., \& Twilt, M. (2017). Isolated arthritis of the temporomandibular joint as the initial manifestation of juvenile idiopathic arthritis. Journal of Rheumatology, 44(11), 1632-1635.

Klenke, D., Quast, A., Prelog, M., Holl-Wieden, A., Riekert, M., Stellzig-Eisenhauer, A., \& Meyer-Marcotty, P. (2018). TMJ pathomorphology in patients with JIA-radiographic parameters for early diagnosis. Head and Face Medicine, 14(1), 1-9.

Kovalko, I., Stoustrup, P., \& Twilt, M. (2018). Temporomandibular Joint Involvement in Juvenile Idiopathic Arthritis: Challenges in Diagnosis, Treatment, and Outcomes. Current Treatment Options in Rheumatology, 4(1), 44-54.

Leksell, E., Hallberg, U., Horne, A., Ernberg, M., \& Hedenberg-Magnusson, B. (2017). Parenting a Child with Juvenile Idiopathic Arthritis, Orofacial Pain, and Dysfunction: A Qualitative Study. Journal of Oral \& Facial Pain and Headache, 31(4), 353-361.

Maliki, A. D., \& Sztajnbok, F. R. (2016). Artrite Idiopática Juvenil: Atualização. Revista Hospital Universitário Pedro Ernesto, 15(2), $140-145$.

Montesinos, G. A., Agudo, R. C. P., Oliveira, A. C. de, James, A. A., Oliveira, J. X., \& Araujo, J. P. (2018). Magnetic resonance imaging changes in pediatric temporomandibular joint: literature review. Clinical and Laboratorial Research in Dentistry, 1-8.

Niibo, P., Pruunsild, C., Voog-Oras, Ü., Nikopensius, T., Jagomägi, T., \& Saag, M. (2016). Contemporary management of TMJ involvement in JIA patients and its orofacial consequences. EPMA Journal, 7(1), 12. https://doi.org/10.1186/s13167-016-0061-7

Okeson, J. P. (2011). Tratamento das desordens temporomandibulares e oclusão. Elsevier.

Pereira, A. S., Shitsuka, D. M., Parreira, F. J., \& Shitsuka, R. (2018). Methodology of cientific research. UFSM. https://repositorio.ufsm.br/bitstream/handle/1/1 5824/Lic_Computacao_Metodologia-Pesquisa-Cientifica.pdf?sequence=1

Piancino, M. G., Cannavale, R., Dalmasso, P., Tonni, I., Garagiola, U., Perillo, L., \& Olivieri, A. N. (2018). Cranial structure and condylar asymmetry of patients with juvenile idiopathic arthritis: a risky growth pattern. Clinical Rheumatology, 37(10), 2667-2673.

Pinto, J. R. R., Pedron, I. G., Utumi, E. R., Miranda, M. E., Pinto, E. C. P., \& Nucci, L. P. (2018). Temporomandibular joint disorders as the only manifestation of juvenile idiopathic arthritis: a case report. Einstein (São Paulo), 16(3), 1-4.

Rigante, D., Bosco, A., \& Esposito, S. (2015). The Etiology of Juvenile Idiopathic Arthritis. Clinical Reviews in Allergy and Immunology, 49(2), 253-261.

Rongo, R., Alstergren, P., Ammendola, L., Bucci, R., Alessio, M., D’Antò, V., \& Michelotti, A. (2019). Temporomandibular joint damage in juvenile idiopathic arthritis: Diagnostic validity of diagnostic criteria for temporomandibular disorders. Journal of Oral Rehabilitation, 46(5), 450-459.

Santos Silveira, O., Cristina Santos Silva, F., Eduardo Neves de Almeida, C., Mesquita Tuji, F., Isaias Seraidarian, P., \& Ricardo Manzi, F. (2014). Use of ct for diagnosing temporomandibular joint. Revista CEFAC, 16(6), 2053-2059.

Stoll, M. L., Amin, D., Powell, K. K., Poholek, C. H., Strait, R. H., Aban, I., \& Waite, P. D. (2018). Risk factors for intraarticular heterotopic bone formation in the temporomandibular joint in Juvenile idiopathic arthritis. Journal of Rheumatology, 45(9), 1301-1307.

Stoor, P., Hodzic, Z., \& Arte, S. (2018). Surgical Treatment of Dentofacial Deformities Caused by Juvenile Idiopathic Arthritis. Journal of Craniofacial Surgery, 29(1), e51-e57.

Stoustrup, P., Kristensen, K. D., Verna, C., Küseler, A., Herlin, T., \& Pedersen, T. K. (2012). Orofacial symptoms related to temporomandibular joint arthritis in juvenile idiopathic arthritis: Smallest detectable difference in self-reported pain intensity. Journal of Rheumatology, 39(12), $2352-2358$. 